\title{
Structural Distortion and Bandgap Increase of Two-Dimensional Perovskites Induced by Trifluoromethyl Substitution on Spacer Cations
}

Pei-Xi Wang, ${ }^{\dagger}$ Amin Morteza Najarian, ${ }^{\dagger}$ Zhaomin Hao, $^{\ddagger}$ Andrew Johnston, ${ }^{\dagger}$ Oleksandr Voznyy, ${ }^{\ddagger}$ Sjoerd Hoogland, ${ }^{\dagger}$ Edward H. Sargent*,+

AUTHOR ADDRESS

tDepartment of Electrical and Computer Engineering, University of Toronto, 10 King's College Road, Toronto, Ontario, M5S 3 G4 Canada

¥Department of Physical and Environmental Sciences, University of Toronto Scarborough, 1065 Military Trail, Scarborough, Ontario, M1C 1A4 Canada

AUTHOR INFORMATION

Corresponding Author

*ted.sargent@utoronto.ca 
a

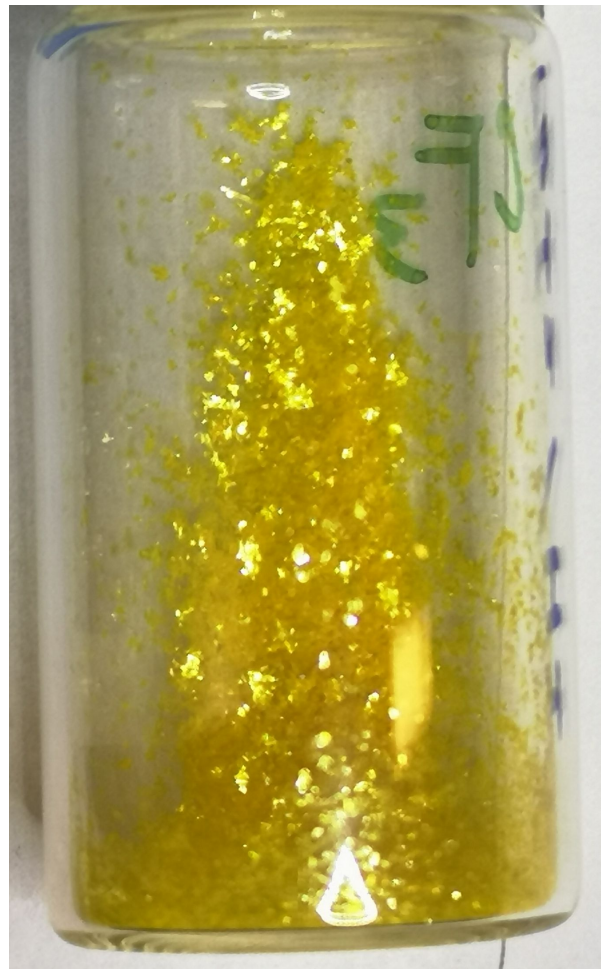

b

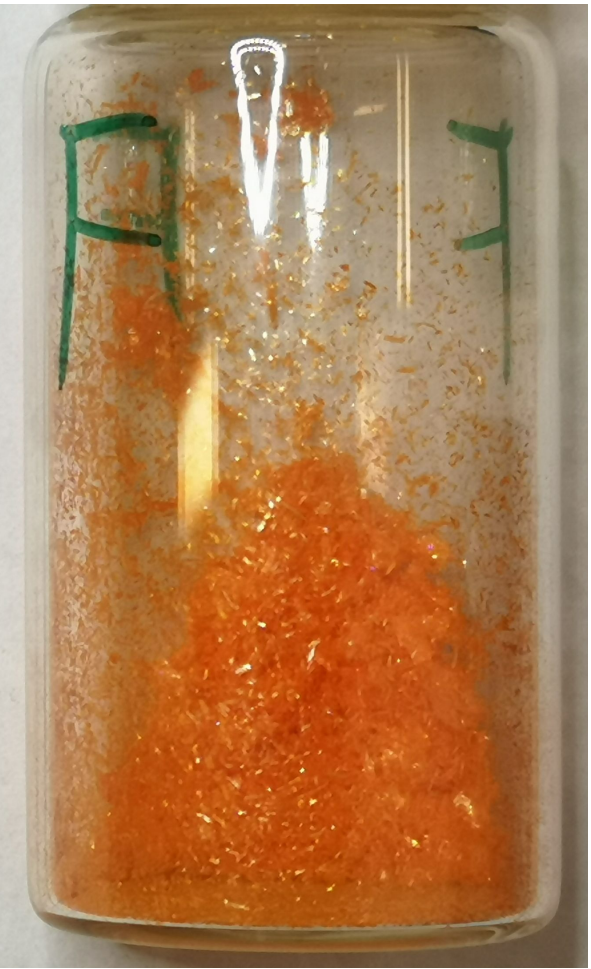

Figure S1. Photographs of $\left(\mathrm{CF}_{3}-\mathrm{PMA}\right)_{2} \mathrm{Pbl}_{4}(\mathbf{a})$ and $(\mathrm{F}-\mathrm{PMA})_{2} \mathrm{Pbl}_{4}(\mathbf{b})$ crystals synthesized in this study. 


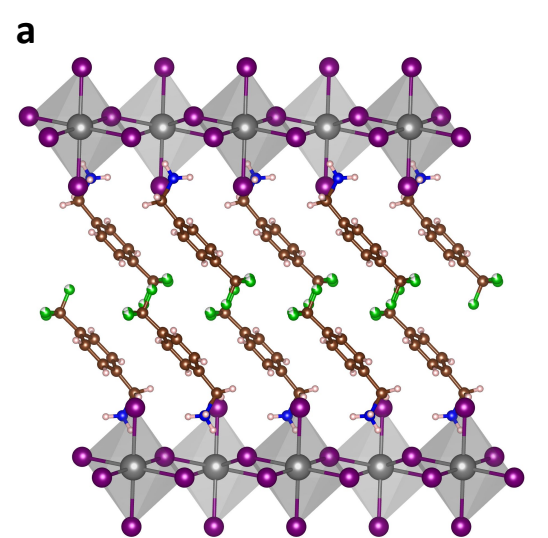

d

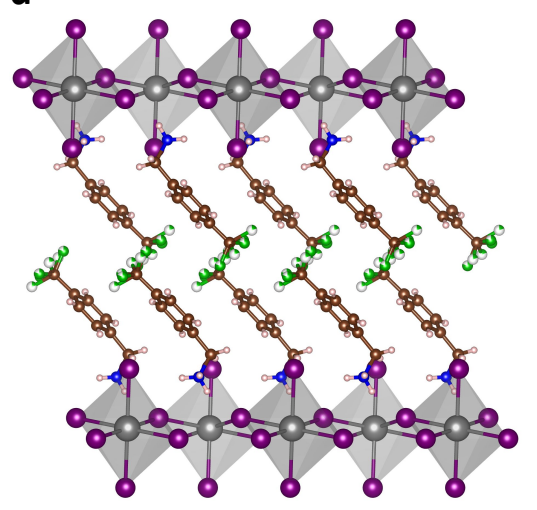

b

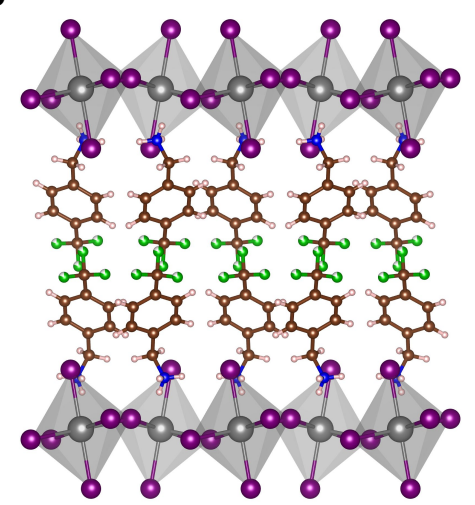

e

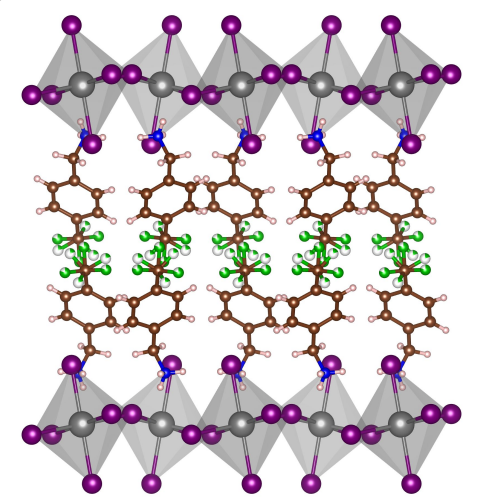

C

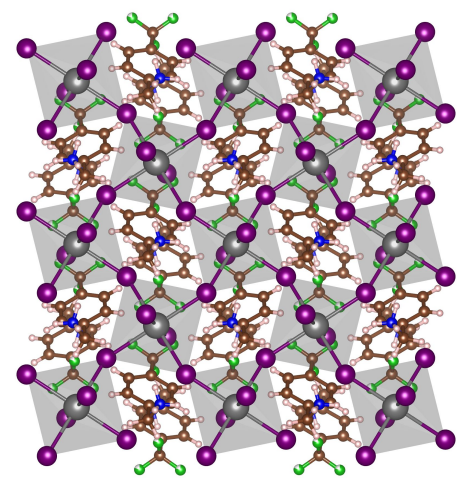

f

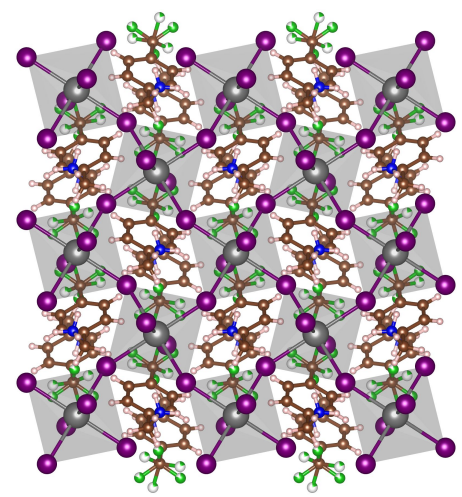

Figure S2. (a-f) Crystal structure of the $\left(\mathrm{CF}_{3}-\mathrm{PMA}\right)_{2} \mathrm{Pbl}_{4}$ perovskite viewed from different directions. The dynamic disorder of trifluoromethyl groups are shown in $\mathbf{d}, \mathbf{e}$, and $\mathbf{f}$. lodide atoms: purple; lead: gray; nitrogen: blue; fluorine: green; carbon: brown; hydrogen: pink. 

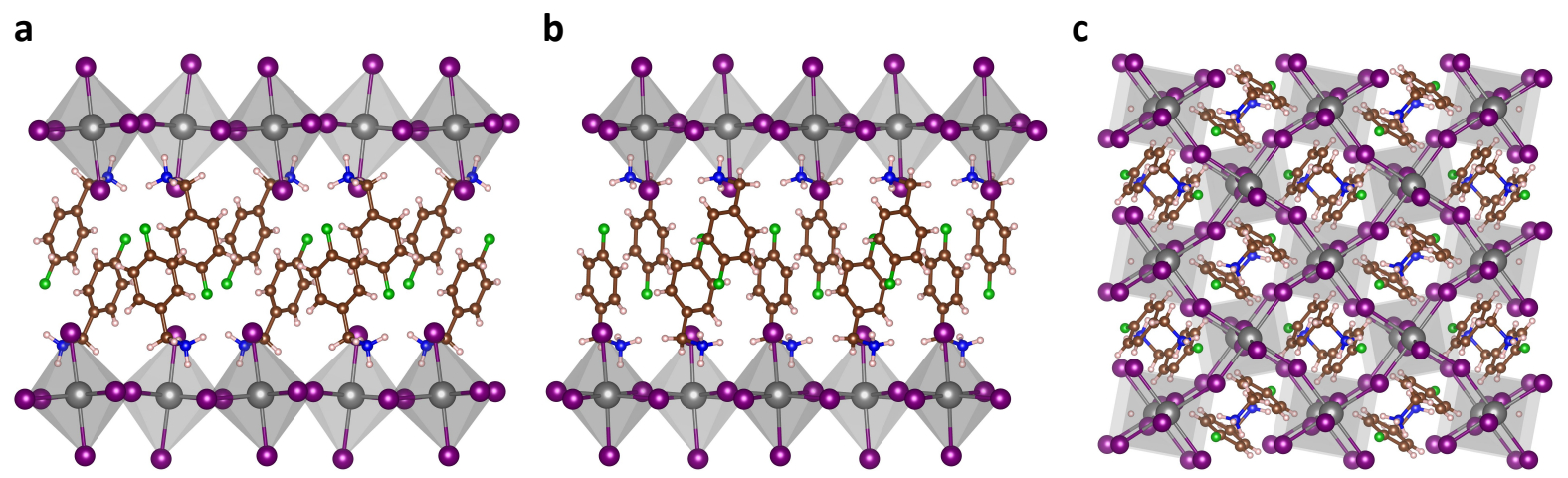

Figure S3. (A-C) Crystal structure of (F-PMA $)_{2} \mathrm{Pbl}_{4}$ perovskite viewed from different directions.

lodide atoms: purple; lead: gray; nitrogen: blue; fluorine: green; carbon: brown; hydrogen: pink. 
a

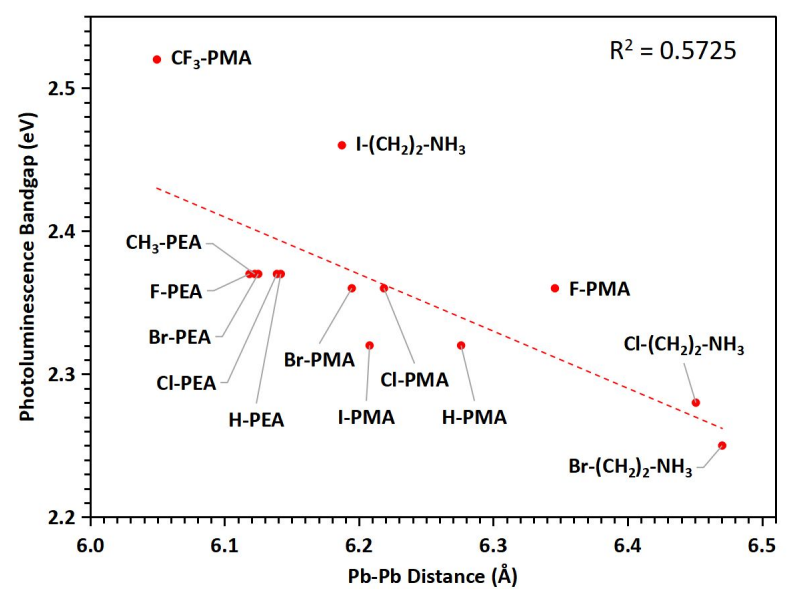

C

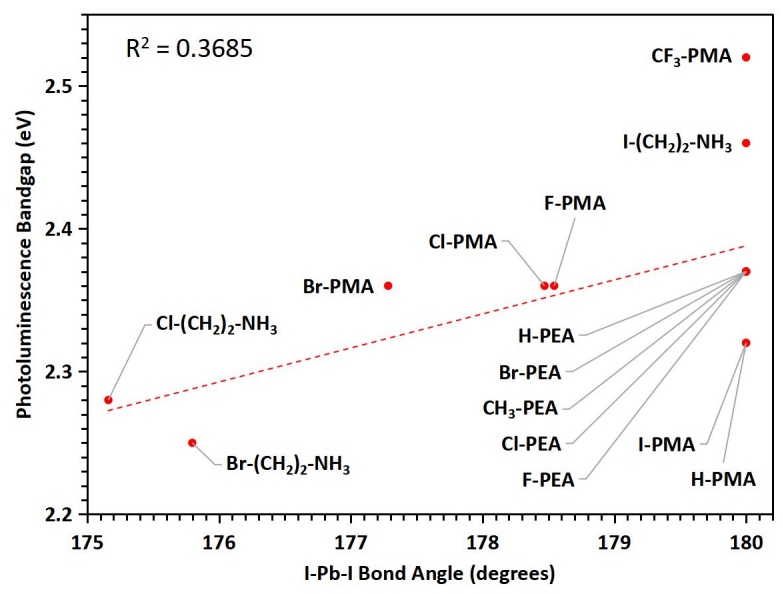

b

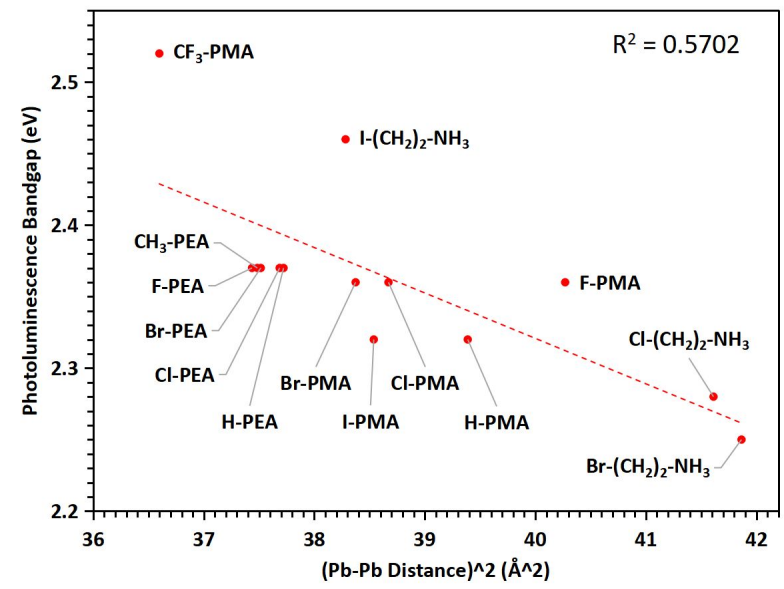

d

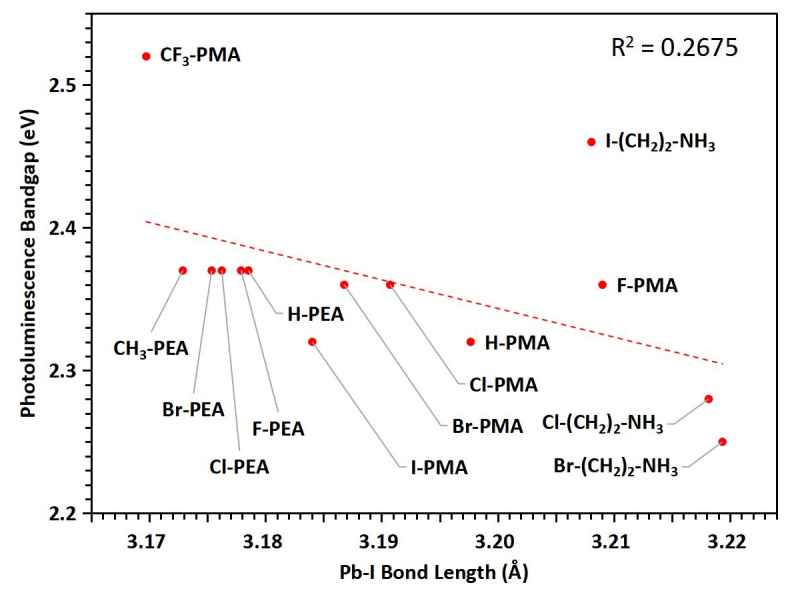

Figure S4. Relationships between the photoluminescence bandgap of two-dimensional lead iodide perovskites and the average $\mathrm{Pb}-\mathrm{Pb}$ distance (a), the squared value of the average $\mathrm{Pb}-\mathrm{Pb}$ distance (b), the average I-Pb-I bond angle (c), or the average $\mathrm{Pb}-\mathrm{I}$ bond length (d). 
Table S1. Comparison of selected structural and optical properties between $\left(\mathrm{CF}_{3}-\mathrm{PMA}\right)_{2} \mathrm{Pbl}_{4}$ and $2 \mathrm{D}$ lead iodide perovskites $\mathrm{A}_{2} \mathrm{Pbl}_{4}$ with other organic spacer cations (the " $\mathrm{A}$ " moieties).

\begin{tabular}{|c|c|c|c|}
\hline Spacer Cation A & PL Bandgap (eV) & I-Pb-I Bond Angle (degrees) & Pb-I Bond Length $(\AA)$ \\
\hline $\mathrm{Br}-\left(\mathrm{CH}_{2}\right)_{2}-\mathrm{NH}_{3}$ & 2.25 & $175.37,175.10,176.92$ & $\begin{array}{c}3.1858,3.1858,3.2679 \\
3.2168,3.2799,3.1799\end{array}$ \\
\hline $\mathrm{Cl}-\left(\mathrm{CH}_{2}\right)_{2}-\mathrm{NH}_{3}$ & 2.28 & $174.26,173.15,178.07$ & $\begin{array}{c}3.2020,3.2020,3.2657 \\
3.2055,3.2616,3.1722\end{array}$ \\
\hline H-PMA & 2.32 & $180.00,180.00,180.00$ & $3.1942,3.2075,3.1913$ \\
\hline I-PMA & 2.32 & $180.00,180.00,180.00$ & $3.2068,3.1815,3.1638$ \\
\hline F-PMA & 2.36 & 179.41, 178.71, 177.51 & $\begin{array}{c}3.1021,3.2303,3.2292 \\
3.2532,3.1993,3.2400\end{array}$ \\
\hline Cl-PMA & 2.36 & $178.11,178.05,178.83$ & $\begin{array}{c}3.1477,3.2447,3.1678 \\
3.2001,3.1955,3.1886\end{array}$ \\
\hline $\mathrm{Br}-\mathrm{PMA}$ & 2.36 & $177.68,177.30,176.87$ & $\begin{array}{c}3.2348,3.1445,3.2107 \\
3.1527,3.1853,3.1927\end{array}$ \\
\hline H-PEA & 2.37 & $180.00,180.00,180.00$ & $3.2063,3.1712,3.1581$ \\
\hline F-PEA & 2.37 & $180.00,180.00,180.00$ & 3.2196, 3.1542, 3.1599 \\
\hline Cl-PEA & 2.37 & $180.00,180.00,180.00$ & 3.2084, 3.1611, 3.1592 \\
\hline $\mathrm{Br}-\mathrm{PEA}$ & 2.37 & $180.00,180.00,180.00$ & $3.2156,3.1573,3.1532$ \\
\hline $\mathrm{CH}_{3}-\mathrm{PEA}$ & 2.37 & $180.00,180.00,180.00$ & $\begin{array}{c}3.2116,3.2116,3.1563 \\
3.1563,3.1499,3.1516\end{array}$ \\
\hline $\mathrm{I}-\left(\mathrm{CH}_{2}\right)_{2}-\mathrm{NH}_{3}$ & 2.46 & $180.00,180.00,180.00$ & $3.1774,3.2243,3.2225$ \\
\hline $\mathrm{CF}_{3}-\mathrm{PMA}$ & 2.52 & $180.00,180.00,180.00$ & $3.1813,3.1620,3.1659$ \\
\hline
\end{tabular}

Note: I-Pb-I bond angles and $\mathrm{Pb}-\mathrm{I}$ bond lengths involving axial (terminal) iodine atoms are in bold numbers. 